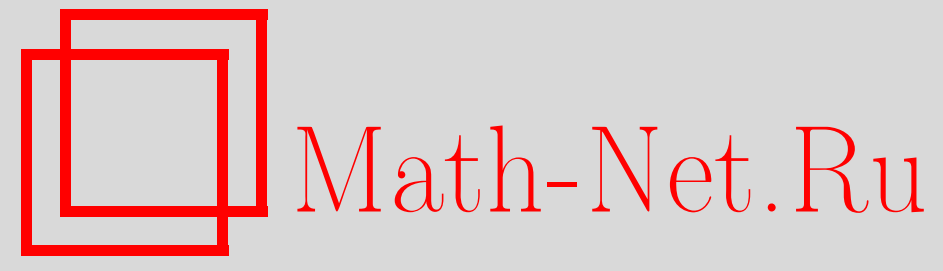

В. Р. Фаталов, Исправления к статье, опубликованной в т. 58 , в. 2, с. 325-354, Теория вероятн. и ее примен., 2014, том 59, выпуск 2, 413-414

DOI: https://doi.org/10.4213/tvp4575

Использование Общероссийского математического портала Math-Net.Ru подразумевает, что вы прочитали и согласны с пользовательским соглашением http://www.mathnet.ru/rus/agreement

Параметры загрузки:

IP : 54.198 .64 .247

26 апреля 2023 г., 03:48:12

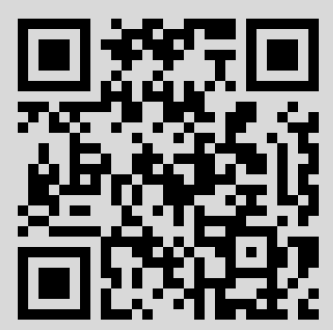




\section{Исправления к статье, опубликованной в т. 58, в. 2, с. 325-354}

Автором обнаружены неточности в формулировках следствия 1 и предложения 2 работы [1]: формула (1.18) содержит ошибочную константу, пункт (ii) предложения 2 содержит ошибочное утверждение. Ниже приведены исправленные варианты соответствующих утверждений.

1. Правильный вариант следствия 1 работы [1] имеет следующий вид.

Следствие 1а. При $u \rightarrow \infty$ выполнень соотношения

$$
\begin{aligned}
& \mathbf{P}\left\{\int_{0}^{1}|\xi(t)| d t>u\right\}=\mu_{\xi}\left\{\int_{0}^{1}|x(t)| d t>u\right\}=\exp \left\{-24 u^{2}\right\} \frac{\sqrt{10}}{\pi}(1+o(1)) \\
& \mathbf{P}\left\{\int_{0}^{1} \xi^{2}(t) d t>u^{2}\right\}=\mu_{\xi}\left\{\int_{0}^{1} x^{2}(t) d t>u^{2}\right\}=2 \exp \left\{-2 \pi^{2} u^{2}\right\}(1+o(1)) .
\end{aligned}
$$

2. Правильный вариант предложения 2 работы [1] имеет следующий вид.

Предложение 2а. (i) Справедливо равенство для норм экстремальных функций, заданных в (3.13):

$$
\left\|x_{\gamma}\right\|_{L^{2}}=\left\|x_{0}\right\|_{L^{2}} \quad \text { для любого } \gamma \in[0,1] .
$$

(ii) Замкнутая компактная кривая $M_{0}$, заданная в (3.14), представляет собой чентрально-симметричное множество, расположенное на сфере радиуса $\left\|x_{0}\right\|_{L^{2}}$ в гильбертовом пространстве $L_{0}^{2}$. Длина кривой $M_{0}$ выражсается формулой

$$
\operatorname{mes}\left(M_{0}\right)=\left\|x_{0}^{\prime}\right\|_{L^{2}}=\frac{4 \sqrt{\pi}}{\sqrt{p(p+2)}}\left(\frac{2+p}{2}\right)^{1 / p} \frac{\Gamma(1 / p)}{\Gamma(1 / p+1 / 2)} .
$$

(iii) Справедливо равенство для норм производных функиий:

$$
\left\|x_{\gamma}^{\prime}(t)\right\|_{L^{2}}=\left\|x_{0}^{\prime}(t)\right\|_{L^{2}} \quad \text { для любого } \gamma \in[0,1] .
$$

Д о к а з а т е л ь с т в о п р е д л о ж е н и я 2а. Все утверждения предложения 2а, кроме формулы (3.44a), доказаны в работе [1]. Докажем формулу (3.44a). Для длины кривой $M_{0}$ в гильбертовом пространстве $L_{0}^{2}$ справедливы следующие соотношения:

$$
\operatorname{mes}\left(M_{0}\right)=\int_{0}^{1}\left\|\frac{\partial}{\partial \gamma} x_{\gamma}(t)\right\|_{L^{2}} d \gamma=\int_{0}^{1}\left\|x_{\gamma}^{\prime}(t)\right\|_{L^{2}} d \gamma=\left\|x_{0}^{\prime}\right\|_{L^{2}} .
$$

Первое равенство в (1) представляет собой несложное обобщение известной формулы длины кривой в конечномерном евклидовом пространстве $\mathbf{R}^{n}$ (см. $[2$, гл. $7, \S 2$, с. 407]) на случай гильбертова пространства $L_{0}^{2}$. Второе равенство в (1) справедливо в силу формулы $(3.13)$ и того факта, что функции $\widehat{x}_{\gamma}(t)$ построены в результате сдвигов на числа $\gamma \in[0,1]$ одной и той же периодической функции $\widehat{x}_{0}(t)$. Третье равенство в $(1)$ вытекает из формулы (3.45). 
Таким образом, первое равенство в формуле (3.44a) доказано. Докажем второе равенство в формуле (3.44a). В силу формул (3.8), (3.38) из [1], для производной экстремальной функции $x_{0}^{\prime}(t)$ выполнены соотношения

$$
\left\|x_{0}^{\prime}\right\|_{L^{2}}^{2}=\lambda_{0} p=\frac{8 \pi}{p}\left(1+\frac{p}{2}\right)^{2 / p-1}\left[\frac{\Gamma(1 / p)}{\Gamma(1 / p+1 / 2)}\right]^{2} .
$$

Отсюда вытекает второе равенство в формуле (3.44a). Предложение 2а доказано.

Д о к а з а т е л ь с т в о с л е д с т в и я 1а. Формула (1.19) доказана в работе [1]. Докажем формулу (1.18). При $p=1$ в силу формулы (3.44a) справедливо равенство mes $\left(M_{0}\right)=4 \sqrt{3}$. Используя это равенство и формулу (4.5) из [1], получаем равенство $c\left(x_{0}\right) /(2 \pi)$ mes $\left(M_{0}\right)=\sqrt{10} / \pi$. Применяя теоремы 1,2 , лемму 11 , формулу $(4.2)$ из [1], получаем, с учетом последнего равенства, соотношение (1.18). Следствие 1а доказано.

\section{СПИСОК ЛИТЕРАТУРЫ}

1. Фаталов В.Р. О методе Лапласа для гауссовских мер в банаховом пространстве. - Теория вероятн. и ее примен., 2013, т. 58, в. 2, с. 325-354.

2. Мищенко A.C., Фоменко A.T. Курс дифференциальной геометрии и топологии. М.: Изд-во Моск. ун-та, 1980, 439 с.

В. Р. Фаталов 